\title{
ARTICLE
}

\section{A longitudinal neurite and free water imaging study in patients with a schizophrenia spectrum disorder}

\author{
Nina Vanessa Kraguljac ${ }^{1}$, Thomas Anthony ${ }^{2}$, William Stonewall Monroe ${ }^{2}$, Frank Michael Skidmore ${ }^{3}$, Charity Johanna Morgan $^{4}$, \\ David Matthew White ${ }^{1}{ }^{1}$, Neel Patel ${ }^{1}$ and Adrienne Carol Lahti ${ }^{1}$
}

Diffusion tensor imaging (DTI) studies show widespread white matter abnormalities in schizophrenia, but it is difficult to directly relate these parameters to biological processes. Neurite orientation dispersion and density imaging (NODDI) is geared toward biophysical characterization of white matter microstructure, but only few studies have leveraged this technique to study white matter alterations. We recruited 42 schizophrenia patients (30 antipsychotic-naïve and 12 currently untreated) and 42 matched controls in this prospective study. We assessed the orientation dispersion index (ODI) and extracellular free water (FW) using singleshell DTI data before and after a 6-week trial of risperidone. Longitudinal data were available for 27 patients. Voxelwise analyses showed significantly increased ODI in the posterior limb of the internal capsule in unmedicated patients $(242$ voxels; $x=-24 ; y=6$; $z=6 ; p<0.01 ; a<0.04$ ), but no alterations in FW. Whole brain measures did not reveal alterations in ODI but a $6.3 \%$ trend-level increase in FW in unmedicated SZ $(t=-1.873 ; p=0.07)$. Baseline ODI was negatively correlated with subsequent response to antipsychotic treatment $(r=-0.38 ; p=0.049)$. Here, we demonstrated altered fiber complexity in medication-naïve and unmedicated patients with a schizophrenia spectrum illness. Lesser whole brain fiber uniformity was predictive of poor response to treatment, suggesting this measure may be a clinically relevant biomarker. Interestingly, we found no significant changes in NODDI indices after short-term treatment with risperidone. Our data show that biophysical diffusion models have promise for the in vivo evaluation of brain microstructure in this devastating neuropsychiatric syndrome.

Neuropsychopharmacology (2019) 44:1932-1939; https://doi.org/10.1038/s41386-019-0427-3

\section{INTRODUCTION}

Diffusion tensor imaging (DTI) studies, which allow the in vivo examination of white matter integrity, show widespread abnormalities in schizophrenia [1]. Importantly, these alterations are associated with disease severity across symptom dimensions [2-5], poor response to antipsychotic treatment [6], and worse overall clinical outcomes $[7,8]$. Findings are often interpreted as evidence of demyelination, decreased oligodendrocyte density, axonal damage, inflammation, or atrophy [9-11], but because conventional diffusion indices are nonspecific, it is difficult to directly relate parameters to biological or pathological processes.

Using the same or slightly varied scan sequences as traditional DTI, advanced diffusion imaging analytics are geared toward biophysical characterization of white matter microstructure. One promising technique is neurite orientation dispersion and density imaging (NODDI), which delineates signal contributions from three tissue compartments [12], where the intraneurite compartment embodies axons and dendrites, the extraneurite compartment represents cell bodies and glial cells, and the nontissue compartment equals cerebrospinal fluid. From this model, neurite orientation dispersion and extracellular free water, which are potentially functionally relevant morphological features of neural tissue, can be derived. While this model typically requires multishell diffusion data, Edwards et al. [13] have recently demonstrated in a healthy control population that the orientation dispersion index (ODI) and extracellular free water (FW) can also be computed with single-shell data.

To aid in interpretation of measures, efforts for histological validation of NODDI have recently been published [14-17]. For example, a study in multiple sclerosis provided unequivocal evidence that the ODI, which assesses the variability of neurite orientation, matches its histological counterpart [15]. Unfortunately, it is not feasible to histologically confirm FW as a proxy of inflammation, as it is an active physiological process that is absent in fixed samples (personal communication with Dr. Gary Zhang). However, because neuroinflammation increases the volume of water in the extracellular space [18] it stands to reason that this would be reflected by a corresponding increase of FW. Lending empirical support to this premise, a longitudinal study in a transgenic Alzheimer model found that the evolution of FW changes corresponds to the inflammatory burden in TgF344-AD rats [19]. Taken together, NODDI imaging could potentially provide novel insights in the pathophysiological processes that underlie white matter abnormalities in neurological and psychiatric disorders.

However, only few studies have assessed these more specific indices in schizophrenia, reporting decreased neurite density in medicated first-episode patients [20], and increased extracellular

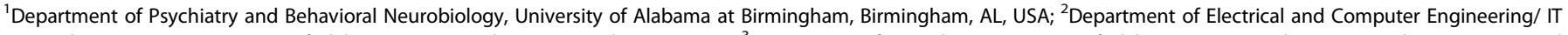

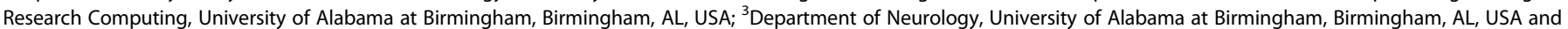
${ }^{4}$ Department of Biostatistics, University of Alabama at Birmingham, Birmingham, AL, USA Correspondence: Nina Vanessa Kraguljac (nkraguljac@uabmc.edu)

Received: 7 February 2019 Revised: 8 May 2019 Accepted: 23 May 2019 Published online: 1 June 2019 
free water in minimally treated first-episode patients [21] and to a lesser extent also in medicated chronic schizophrenia patients [22]. Results are suggestive of altered neurite morphology and neuroinflammation as pathophysiological processes in the illness, but because all studies enrolled medicated patients, it is not clear to which extent these deviations can be attributed to antipsychotic medication effects as opposed to intrinsic illness characteristics. In cross-sectional studies, advanced diffusion indices were not correlated with antipsychotic doses [20] or medication status [21], suggesting that dopamine D2 receptor blockers may not affect these markers. However, this has never directly been tested in a longitudinal design, which is especially important as longitudinal studies have shown that traditional diffusion tensor imaging markers may be sensitive to antipsychotic medication exposure $[6,23]$.

The goal of this study was to apply NODDI to investigate white matter ODI and FW in unmedicated patients with a schizophrenia spectrum illness (SZ) and to assess the effects of 6 weeks treatment with risperidone on these measures. We hypothesized that ODI and FW would be altered in unmedicated SZ compared to matched healthy controls $(\mathrm{HC})$, but that short-term treatment with antipsychotic medications would not affect measurements. In an exploratory fashion, we also investigated the relationships between these indices and symptom severity as well as antipsychotic treatment response. Because a recent study reported that greater FW predicted better neurocognitive function in first-episode psychosis patients, we also explored the relationship between NODDI indices and cognition [21].

\section{MATERIALS AND METHODS}

We recruited SZ from the outpatient, inpatient, and emergency services at the University of Alabama at Birmingham (UAB). HC matched on age, sex, and parental occupation were recruited by advertisements. Written informed consent was obtained prior to enrollment after subjects were deemed to have capacity to provide consent [24] in this UAB Institutional Review Board approved study.

Diagnoses were established through consensus of two board certified psychiatrists ( $A C L$ and NVK) taking diagnostic interview for genetic studies and medical records into consideration as available. The brief psychiatric rating scale (BPRS) and repeatable battery for the assessment of neuropsychological status were used to assess symptom severity and cognition respectively. Subjects were excluded if they had major neurological or medical conditions, history of head trauma with loss of consciousness, substance use disorders (excluding nicotine) within 6 months of imaging, were prescribed medications known to affect brain function, were pregnant or breastfeeding, or had MRI contraindications. Controls with a personal or family history in a firstdegree relative of a psychotic disorder were excluded.

We enrolled $42 \mathrm{SZ}$ who were antipsychotic-naïve or off antipsychotic medications for at least 2 weeks (determined by self report) in a 6-week trial of risperidone using a flexible dosing regimen. Medications were prescribed by $\mathrm{ACL}$ and NVK. Risperidone was started at 1-3 mg and titrated in 1-2 mg increments, dosing was based on therapeutic and side effects. Use of adjuvant medications was permitted as indicated. Compliance was monitored with pill counts at each visit. Scans were acquired prior to treatment and after 6 weeks of treatment, nine SZ dropped out before study completion.

We also enrolled $42 \mathrm{HC}, 38$ of those were scanned twice, on average $51.5 \pm 19.8$ days apart.

\section{Data acquisition}

Imaging was done on a $3 \mathrm{~T}$ head-only scanner (Siemens Magnetom Allegra) using a circularly polarized transmit/receive head coil. A T1-weighted scan was acquired for anatomical reference (MPRAGE, TR/TE/TI = 2300/3.93/1100 ms, $1 \mathrm{~mm}$ isotropic voxels). Two diffusion-weighted runs were acquired [30 directions, $b=1000 \mathrm{~s} / \mathrm{mm}^{2}$, TR/TE $=9200 / 96 \mathrm{~ms}$, field of view $=246 \times$ $246 \mathrm{~mm}$, matrix $=112 \times 112,60$ slices, interleaved acquisition, 2.2 $\mathrm{mm}$ isotropic voxel size]. Runs were concatenated to increase the signal-to-noise ratio (for three SZ datasets only one run was acquired). Five images with no diffusion gradients (b0; $b=0 \mathrm{~s} / \mathrm{mm}^{2}$ ) were also acquired.

\section{Data processing}

The data processing pipeline is illustrated in Fig. 1. Preprocessing was performed in TORTOISE (version 2.5.2) [25], as previously described [26]. Briefly, this included correction for artifacts due to bulk motion, eddy currents and susceptibility artifact, resampling of diffusion images to $1.5 \mathrm{~mm}$ isotropic voxels, and rotation of gradient tables. Five datasets were processed without the anatomical reference image, because the reference image was not available or of poor quality resulting in miss-registration. For calculation of ODI and FW maps, we used the NODDI toolbox (version 1.0) [12]. To spatially normalize images to the Illinois Institute of Technology atlas (IIT2) space, we implemented an optimized nonlinear image registration procedure using a modified version of 3dQwarp in AFNI [27].

Data quality control

We assessed bulk motion and excluded datasets with an average root mean square of absolute displacement $\left(\mathrm{RMS}_{\mathrm{abs}}\right)$ of greater than half the voxel edge length $(1.1 \mathrm{~mm})$ or root mean square of relative displacement $\left(\mathrm{RMS}_{\text {rel }}\right)$ of $>0.05 \mathrm{~mm}$ per run from further analyses [28]. One SZ baseline and one SZ week six datasets were rejected based on these criteria.

We visually inspected both raw data and NODDI maps for artifacts and anomalies in diffusion parameters. Based on this, ten datasets were excluded from further analysis (one $\mathrm{HC}$ and three SZ baseline as well as two HC and four SZ week 6 scans).

Final analyses included good quality datasets from $41 \mathrm{HC}$ and 38 SZ at baseline, and $36 \mathrm{HC}$ and $28 \mathrm{SZ}$ at week 6 . A total of $36 \mathrm{HC}$ and 27 SZ had good quality datasets for both baseline and week 6 scans.

\section{Statistics}

To examine whole brain voxelwise group differences and changes with antipsychotic treatment in diffusion indices within a IIT2 based white matter mask we used $\mathrm{AFNI}^{\prime} \mathrm{s} 3 \mathrm{dt}$ test++; a priori defined co-variates included age and gender. Because baseline head motion differed between groups $\left(\mathrm{RMS}_{\mathrm{abs}}\right.$ baseline: $\mathrm{HC} 0.56 \pm$ $0.10 \mathrm{~mm} ; \mathrm{SZ} 0.62 \pm 0.14 \mathrm{~mm} ; t=-2.18 ; p=0.03 ; \mathrm{RMS}_{\text {abs }}$ week 6 : $\mathrm{HC} 0.56 \pm 0.10 \mathrm{~mm} ; \mathrm{SZ} 0.62 \pm 0.16 \mathrm{~mm} ; t=-1.83 ; p=0.09 ; \mathrm{RMS}_{\text {rel }}$ baseline: $\mathrm{HC} 0.14 \pm 0.002 \mathrm{~mm}$; SZ $0.17 \pm 0.005 \mathrm{~mm} ; t=-3.24 ; p=$ 0.002; $\mathrm{RMS}_{\text {rel }}$ week 6: HC $0.15 \pm 0.03 \mathrm{~mm}$; SZ $0.16 \pm 0.05 \mathrm{~mm} ; t=$ $-1.33 ; \mathrm{p}=0.19$ ) we included $\mathrm{RMS}_{\text {rel }}$ as additional covariate in analyses. Clustsim (10,000 iterations) was run to control for the false-positive rate (voxelwise $p=0.01$; cluster threshold $a=0.05$ ) $[29,30]$.

As a post hoc analyses, we extracted whole brain white matter ODI and FW for each subject at each time-point and compared baseline group differences using two-sample $t$ tests and changes over time in SZ using paired-sample $t$ tests. In an exploratory fashion, we also examined partial correlations between whole brain white matter diffusion metrics clinical measures in SZ (treatment response was defined as follows: [BPRS total baseline - BPRS total week 6]/BPRS total at baseline $\times 100$; consistent with the definition of treatment response we used in a number of prior publications [31-33]), controlling for age, sex, and $\mathrm{RMS}_{\text {rel. }}$.

\section{RESULTS}

HC and SZ did not differ in gender, age, or parental socioeconomic status (Table 1). BPRS scores significantly decreased after 6 weeks 


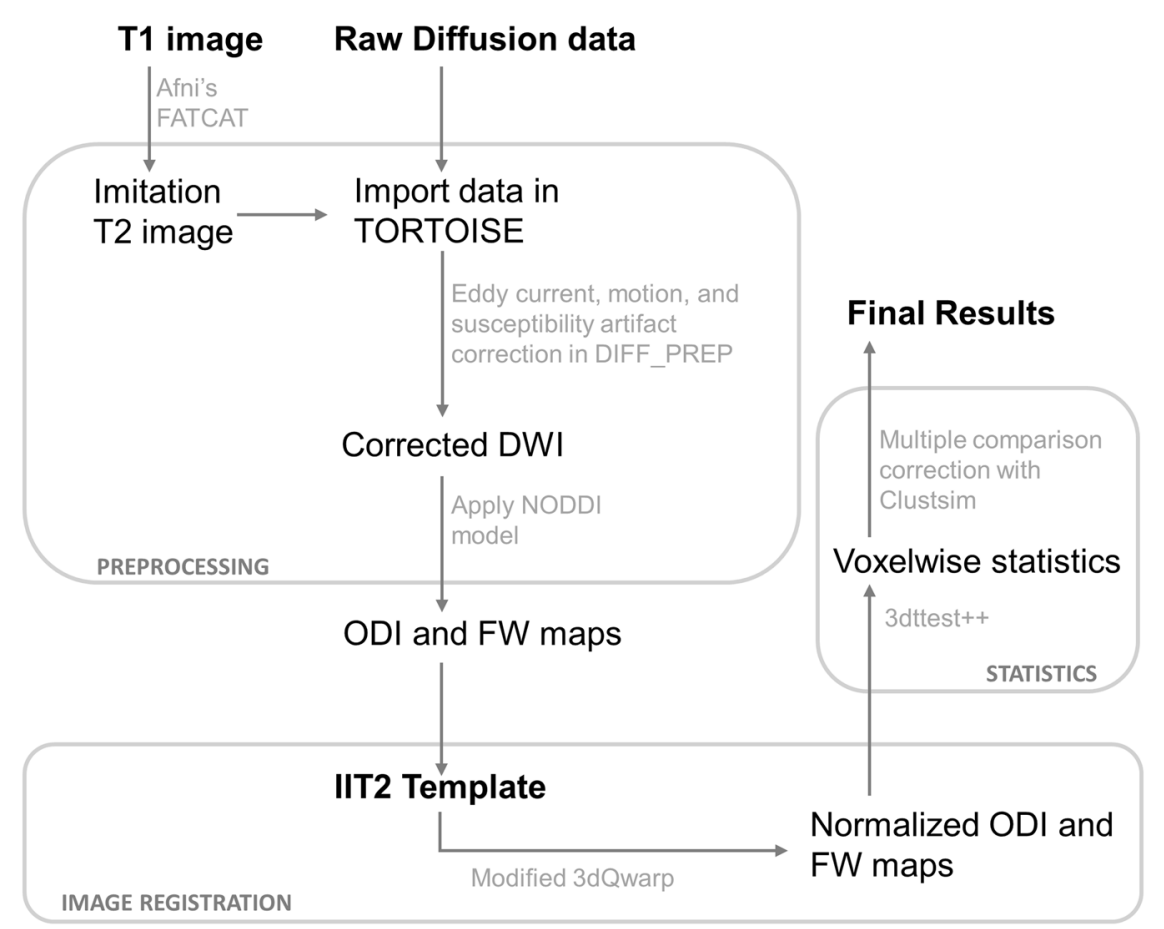

Fig. 1 Schematic of the diffusion data processing pipeline. In preparation for DTI image registration, T1 images were processed through FATCAT in AFNI to create a volume with an approximate T2-weighted contrast. This "imitation T2" image was used solely for providing an anatomical reference. Artifacts due to bulk motion, eddy currents, and EPI susceptibility-induced geometric distortions were removed using DIFF_PREP with a single interpolation step. Prior to registration, diffusion weighted, and structural images were upsampled at a factor two and smoothed with a Perona-Malik filter to compute the transformations which were then applied to original images. Bspline correction was done using the imitation T2 image. For calculation of orientation dispersion and extracellular free water maps, we used the NODDI toolbox. For nonlinear image registration, we used a modified version of 3dQwarp in AFNI which implements an iterative refinement, where an input image is repeatedly processed through an optimizer with a final patch size of $5 \mathrm{~mm}^{3}$. AFNI's 3dt test++ was implemented for statistical analyses, using Clustsim for multiple comparison corrections. Abbreviations: DWI diffusion weighted image, NODDI neurite orientation dispersion and density imaging, FW extracellular free water, ODI orientation dispersion index

of treatment with risperidone (average daily dose at endpoint was $3.73 \pm 1.72 \mathrm{mg}$ ).

NODDI abnormalities in unmedicated SZ

Voxelwise analyses comparing unmedicated SZ to HC showed one area of significant increase in ODI, the posterior limb of the internal capsule (cluster extent: 242 voxels; talairach coordinates for the center mass: $x=-24 ; y=6 ; z=6 ; p<0.01 ; a<0.04$; Fig. 2). This was consistent with a repeat analysis including only antipsychotic-naïve SZ and matched $\mathrm{HC}$ also showing an increase in ODI in the posterior limb of the internal capsule (cluster extent: 413 voxels; $x=-25 ; y=6 ; z=13 ; p<0.01 ; a<0.02$ ). Examining FW using voxelwise analyses, we did not find significant alterations baseline in SZ compared to HC; this was also true when only antipsychotic-naïve SZ and their matches were included in analyses.

Post hoc analyses measuring whole brain white matter diffusion indices did not reveal alterations in whole brain ODI $(\mathrm{d} f=78 ; t=$ $-0.961 ; p=0.34$ ), but showed a $6.3 \%$ increase in whole brain FW in unmedicated SZ which was significant at trend level $(\mathrm{d} f=78$; $t=-1.873 ; p=0.07$; Fig. 3a).

NODDI changes after 6 weeks of treatment in SZ

We failed to detect a change in ODI or FW in patients after 6 weeks of treatment with risperidone with voxelwise analyses. Again, the results were the same when only antipsychotic-naïve SZ were included. We also did not see changes in whole brain measures after treatment (ODI: $\mathrm{d} f=26 ; t=0.136 ; p=0.89 ; \mathrm{FW}: \mathrm{d} f=26 ; t=0.526$, $p=0.60$; Fig. 3b).
Longitudinal NODDI in HC

Voxelwise diffusion indices did not differ between baseline and week six in $\mathrm{HC}$, even when lowering the statistical threshold to $p_{\text {uncorrected }}<0.001$. Coefficients of variation were $2.2 \%$ for wholebrain ODI and $4.9 \%$ for whole-brain FW, suggesting that these measurements are stable across time.

Relationship between NODDI and clinical variables Exploratory analysis demonstrate a negative correlation between baseline ODI and treatment response both in the whole sample $(r=-0.38 ; p=0.049 ;$ Fig. 4$)$, and when only medication-naïve subjects were taken into consideration $(r=-0.52 ; p=0.027)$. We found no significant correlations between ODI in the posterior limb of the internal capsule and the "motor retardation" or "mannerisms and posturing" items of the BPRS. No other relationships between diffusion indices and positive, negative, or cognitive symptom severity, or duration of untreated psychosis were seen (all $p>0.05$ ).

\section{DISCUSSION}

To our knowledge, this is the first prospective, longitudinal study applying NODDI, a biophysical diffusion model, to characterize white matter integrity in unmedicated schizophrenia spectrum disorder patients and to assess the effects of short-term treatment with risperidone. Our results demonstrate increased ODI in the posterior limb of the internal capsule and a $6.3 \%$ increase in whole brain FW in unmedicated patients which was significant at trend level and indicates that abnormalities are not merely a confound 
Table 1. Demographics and clinical measures ${ }^{\mathrm{a}}$

\begin{tabular}{|c|c|c|c|c|}
\hline & $S Z(n=42)$ & $\mathrm{HC}(n=42)$ & $t / X^{2}$ & $p$ Value \\
\hline Gender (\% male) & 61.9 & 61.9 & 0.00 & 1.0 \\
\hline Age & $26.62(9.00)$ & $27.88(9.43)$ & 0.63 & .53 \\
\hline Parental Occupation ${ }^{\text {b }}$ & $7.16(6.13)$ & $5.45(4.18)$ & 16.28 & .30 \\
\hline Smoking (packs per day) & $0.38(0.53)$ & $0.23(0.43)$ & -1.47 & .15 \\
\hline \multicolumn{5}{|l|}{ Diagnosis } \\
\hline Schizophrenia & 34 & & & \\
\hline Schizoaffective Disorder & 5 & & & \\
\hline Brief Psychotic Disorder & 3 & & & \\
\hline Illness Duration (years) ${ }^{c}$ & $15.00(8.67)$ & & & \\
\hline \multicolumn{5}{|l|}{$\begin{array}{l}\text { Prior antipsychotic } \\
\text { treatment }\end{array}$} \\
\hline $\begin{array}{l}\text { Antipsychotic naïve } \\
\text { (yes/ no) }\end{array}$ & $30 / 12$ & & & \\
\hline $\begin{array}{l}\text { Antipsychotic free } \\
\text { interval (months) }^{c}\end{array}$ & $17.33(36.91)$ & & & \\
\hline \multicolumn{5}{|l|}{$B P R S^{\mathrm{d}}$} \\
\hline \multicolumn{5}{|l|}{ Total } \\
\hline Baseline & $50.26(9.30)$ & & & \\
\hline Week $\sigma^{e}$ & $32.36(9.60)^{9}$ & & & \\
\hline \multicolumn{5}{|l|}{ Positive } \\
\hline Baseline & $10.93(3.46)$ & & & \\
\hline Week $6^{\mathrm{e}}$ & $5.24(2.55)^{\mathrm{h}}$ & & & \\
\hline \multicolumn{5}{|l|}{ Negative } \\
\hline Baseline & 7.19 (3.36) & & & \\
\hline Week $6^{\mathrm{e}}$ & $5.73(2.41)^{\mathrm{i}}$ & & & \\
\hline \multicolumn{5}{|l|}{$\operatorname{RBANS}^{\mathrm{f}}$} \\
\hline Total index & $71.82(14.37)$ & $89.67(12.73)$ & 5.89 & $<0.01$ \\
\hline Immediate memory & $77.42(16.35)$ & $97.07(15.33)$ & 5.55 & $<0.01$ \\
\hline Visuospatial & $73.24(16.52)$ & $82.48(15.41)$ & 2.59 & 0.01 \\
\hline Language & $85.50(12.82)$ & $96.64(14.04)$ & 3.76 & $<0.01$ \\
\hline Attention span & 80.39 (19.67) & $94.07(18.45)$ & 3.21 & $<0.01$ \\
\hline Delayed memory & $71.79(20.57)$ & $91.38(10.13)$ & 5.32 & $<0.01$ \\
\hline
\end{tabular}

$S Z$ schizophrenia, $H C$ healthy control

${ }^{a}$ Mean (SD) unless indicated otherwise

${ }^{\mathrm{b}}$ Ranks determined from Diagnostic Interview for Genetic Studies (1-18 scale); higher rank (lower numerical value) corresponds to higher socioeconomic status

'Only patients who are not antipsychotic naïve $(n=12)$, illness duration since first diagnosis

dBrief psychiatric rating scale (1-7 scale); positive (conceptual disorganization, hallucinatory behavior, and unusual thought content); negative (emotional withdrawal, motor retardation, and blunted affect)

$\mathrm{e}_{n}=33$

fRepeatable battery for the assessment of neuropsychological status

${ }^{g} t=11.19 ; p<0.01$

$\mathrm{h}_{t}=9.45 ; p<0.01$

${ }^{i} t=2.98 ; p<0.01$

of antipsychotic treatment. Interestingly, higher whole-brain ODI at baseline was associated with poorer response to subsequent antipsychotic treatment, suggesting that fiber orientation is a clinically relevant microstructural white matter feature.

Here, we found increased ODI in the posterior limb of the internal capsule in patients. In transparent mouse brains ODI has been shown to be higher in areas of fiber crossing compared to areas of parallel fibers [16] and microscopic mapping in postmortem human brains demonstrated that $\mathrm{ODI}$ is largely
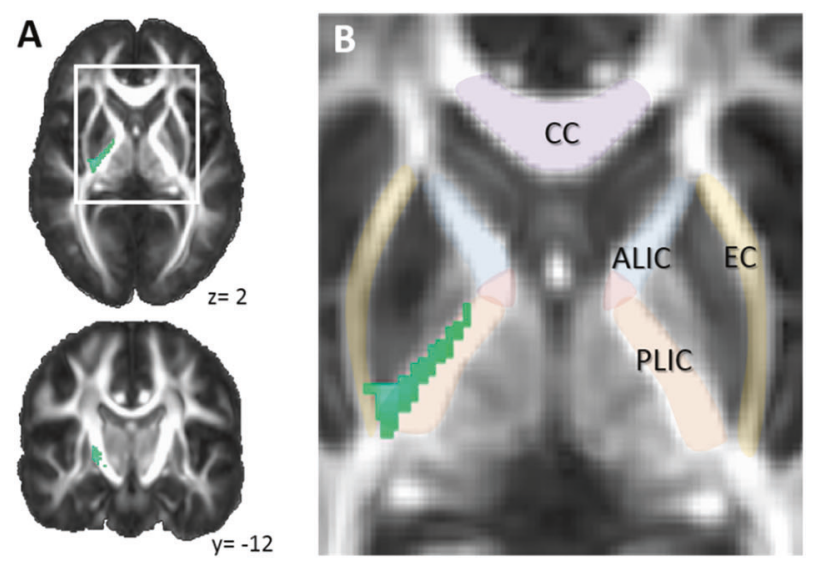

$\mathrm{SZ}>\mathrm{HC}=\mathrm{HC}>\mathrm{SZ}$

C

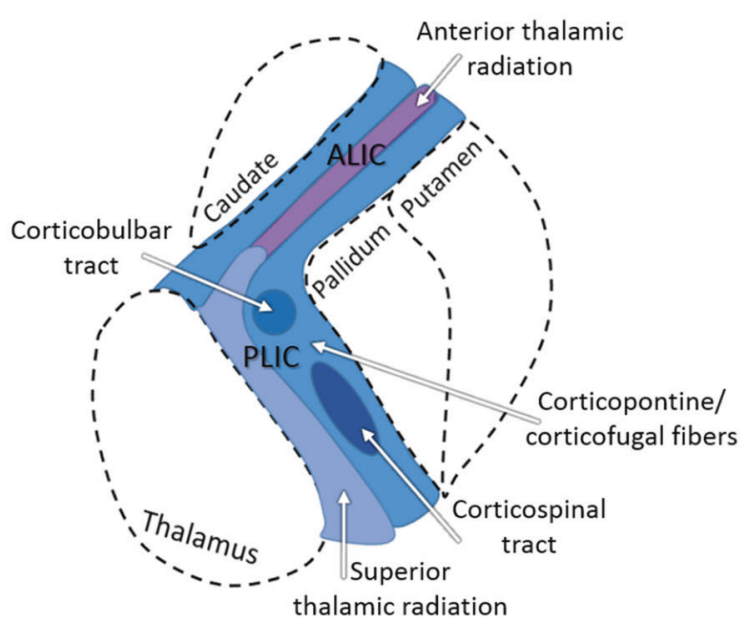

Fig. 2 Results of voxelwise statistics. a. Orientation dispersion in the posterior limb of the internal capsule in unmediated patients with schizophrenia is increased compared to healthy controls. Numbers adjacent to slices indicate $x$ and $y$ coordinates in Talairach convention. b Zoomed image of area of increased orientation dispersion in patients and surrounding white matter structures. Color bar indicates $z$ scores. c Illustration of an axial section depicting the fiber architecture of the anterior and posterior limb of the internal capsule. Abbreviations: ALIC anterior limb of the internal capsule, CC corpus callosum, EC external capsule, PLIC posterior limb of the internal capsule

explained by the signal stemming from myelinated axons while the astrocytic contribution appears negligible [34]. In other words, a greater ODI seen in our study can be interpreted as lesser fiber uniformity in this area. This was previously reported in medicated first-episode and chronic schizophrenia patients using a different diffusion based assessment, constrained spherical deconvolution [35]. Decreased local coherence of fiber orientation has also been detected in the corpus callosum, cingulum, internal capsule, and fornix in chronic schizophrenia [36]. While the only other study using ODI to quantify white matter fiber complexity in schizophrenia spectrum disorders found no abnormalities in medicated first-episode patients compared to controls, they did observe that worse fiber organization with greater age in the corpus callosum in patients [20], suggesting this might be a progressive abnormality.

A number of studies have identified abnormalities in the size, symmetry, and diffusivity of the internal capsule in schizophrenia [37-40]. In parallel, motor behavior abnormalities have been reported in the illness [41], which have been linked to structural 
A
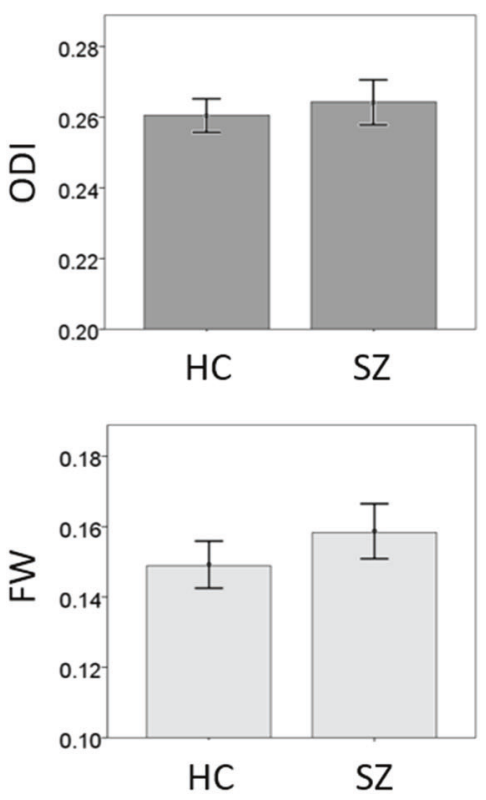

B

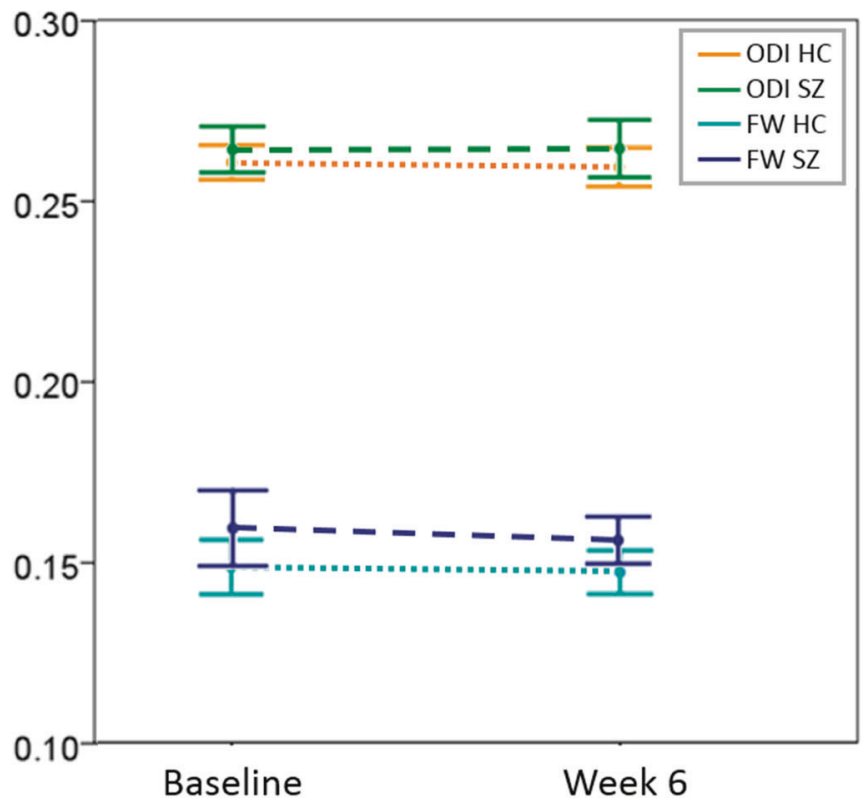

Fig. 3 Whole-brain orientation dispersion and extracellular free water. a Whole-brain NODDI measures in unmedicated patients with schizophrenia and healthy controls. Orientation dispersion does not differ between groups, extracellular free water is increased by $6.3 \%$ in patients compared to controls which is significant at trend-level $(p=0.07)$. Error bars depict the $95 \%$ confidence interval. b Time course of NODDI measures. No change in whole brain orientation dispersion or extracellular free water was observed in patients with schizophrenia or in healthy controls. Error bars indicate $95 \%$ confidence intervals. Abbreviations: FW extracellular free water, ODI orientation dispersion index (ODI is a value between zero and one that describes the spatial configuration of the neurite structures, with high values of ODI corresponding to highly dispersed neurites and small values corresponding to highly aligned axons), HC healthy control, SZ patient with schizophrenia

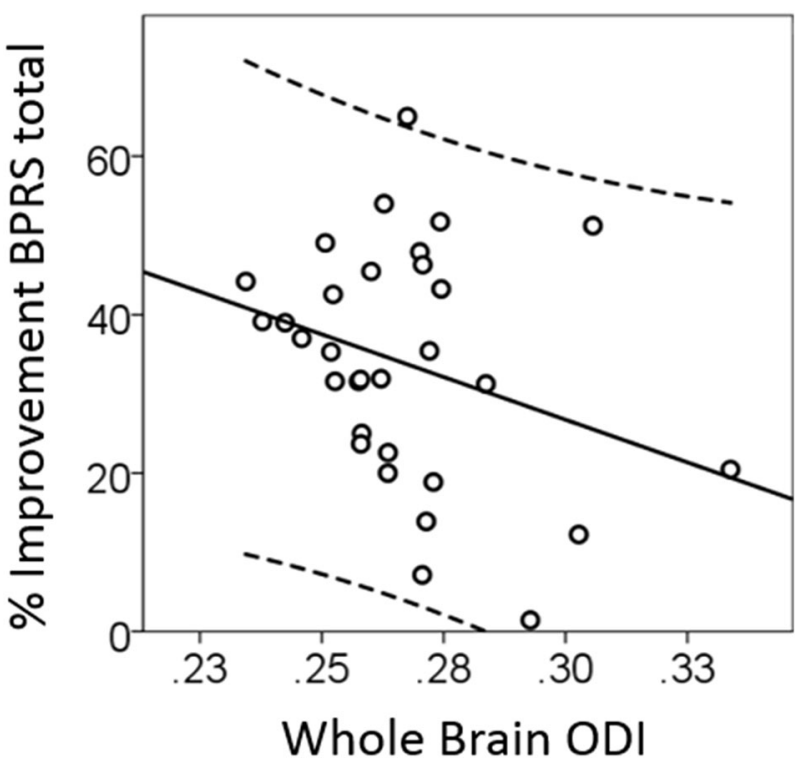

Fig. 4 Whole-brain orientation dispersion in unmedicated patients with schizophrenia predicts subsequent response to risperidone $(r=-0.38 ; p=0.049)$. Abbreviations: BPRS brief psychiatric rating scale, ODI orientation dispersion index

and connectivity abnormalities between the basal ganglia, thalamus, and motor cortex implicating the internal capsule in motor pathology [42-44]. To our knowledge, the first to report evidence of lesser uniformity of fibers in this region were Buchsbaum et al. [45] who used path length as a proxy for fiber organization. Investigations commonly focus on the anterior limb, which contains the anterior thalamic radiation, a fiber tract that connects the thalamus and prefrontal cortex and has been linked to thalamo-cortical dysconnectivity in the illness [46]. Nonetheless, some studies also implicate the posterior limb, which contains corticospinal, cortico-bulbar, and cortico-cerebellar fibers and the superior thalamic radiation in the schizophrenia pathology. Posterior limb white matter integrity reductions, measured as fractional anisotropy, were previously reported in a small group of patients with early-stage schizophrenia [47]. Further underscoring the role of this region in the illness, a machine learning based pattern recognition study discovered that fractional anisotropy of the corticospinal tract was one of the most prominent discriminant features separating $\mathrm{HC}$ from first-episode schizophrenia patients [48]. Interestingly, the posterior limb of the internal capsule was one of the few white matter regions where no fractional anisotropy abnormalities were detected in chronic schizophrenia patients in a large individual data meta-analysis in conducted by the ENIGMA DTI working group [1]. It is possible that ODI and fractional anisotropy capture distinct aspects of microstructural pathology. Alternatively, because delayed maturation of the corticospinal tract resulting in diffusion abnormalities in first-episode psychosis becomes less prominent as the illness progresses [49], differences in disease stages could explain discrepancies in findings.

At baseline, greater whole brain ODI, indicative of lesser fiber uniformity, was predictive of poorer response to subsequent antipsychotic treatment, both in the entire group and in the subset of patients who were antipsychotic-naïve at the time of enrollment. This is consistent with a recent report by Reis-Marques et al. [6] who found widespread fractional anisotropy decreases in first-episode patients with a subsequent poor response to antipsychotic treatment compared to those with a good response. Similarly, first-episode psychosis patients who had greater white 
matter integrity deficits in the superior longitudinal fasciculus and uncinate fasciculus would more likely be classified as poor treatment responders 6 months later [7]. On a connectome level, baseline global white matter network organization showed greater alterations in first-episode psychosis patients who subsequently showed poor antipsychotic treatment response compared to those with good response [50]. Like us, they found no regionally specific white matter changes predicting treatment response, but whole brain white matter organization did have predictive value. In sum, evidence supports that white matter signatures could be leveraged as clinically relevant biomarkers, where more intact white matter is predictive favorable response to antipsychotic treatment.

Examining $\mathrm{FW}$ in unmedicated patients, we did not find regional alterations in voxelwise analyses, but a $6.3 \%$ increase in whole-brain FW in unmedicated patients compared to controls that was significant at trend level. Only a few prior studies have investigated FW in schizophrenia spectrum patients. One reported a global FW increase in minimally treated firstepisode patients [21], while others reported elevated FW in a limited number of brain regions in medicated chronic patients $[22,51]$. Even though all interpreted their findings as evidence of neuroinflammation, the literature points towards a complex process that may differ across illness stages. For example, several positron emission tomography studies using TSPO as tracer examined evidence of neuroinflammation in psychosis spectrum patients. They found activation of microglia in gray matter in medicated recent onset schizophrenia patients [52] and in the hippocampus in acutely psychotic patients [53]. However, others did not find alterations in glial markers in medicated chronic patients [54, 55]. These variable findings across studies could be interpreted as evidence of a dynamic imbalance of inflammatory pathways that may be most prominent in the early stages of the illness and/or during acute psychotic episodes.

It is notable that that the magnitude of FW excess in patients we detected is smaller than that of a prior study that found wholebrain white matter FW values where approximately $11 \%$ higher in patients compared to controls [56]. Because prior studies reconstructed FW maps with a two compartment model optimized specifically for FW detection [57] as opposed to NODDI which assesses microstructural tissue properties based on a three compartment model, it is possible that they have had greater precision for detection pathological FW increases.

Our findings further extend the existing literature by demonstrating, for the first time in a prospective design, that antipsychotic treatment with risperidone did not affect ODI or FW, both at the voxel and at the whole-brain level. This is consistent with cross-sectional studies reporting that biophysical diffusion indices are not correlated with antipsychotic doses [20] or medication status [21], suggesting that dopamine D2 receptor blockers may not affect these markers. However, we acknowledge that it is possible that the chosen timeframe was too short to observe antipsychotic treatment related changes in ODI or FW. The time to clinical response to antipsychotic treatment in firstepisode psychosis has been shown to be up to 16 weeks [58]; it thus is conceivable that changes in NODDI parameters may become evident only after longer treatment timeframes. Prospective studies following first-episode psychosis patients over the course of months or years are warranted to map the evolution of antipsychotic medication effects on white matter microstructure and will be pivotal in resting the debate as to antipsychotic medications being neuroprotective, detrimental, or merely a confound in the assessment of progressive brain changes in the illness.

Several strengths and limitations need to be considered in the interpretation of our data. We enrolled unmedicated SZ, the majority of which had no prior antipsychotic medication exposure, used a single-antipsychotic medication, and carefully matched groups on demographic characteristics to minimize variance in the data. We excluded subjects with major neurological or medical conditions, but did not record nonpsychotropic medications prescribed at the time of enrollment. It is, therefore, possible that some patients were prescribed medications that could affect brain microstructure. We obtained single-shell diffusion data to characterize ODI and FW with NODDI, a technique that typically uses multi-shell diffusion data. It has recently been demonstrated in healthy volunteers that ODI and FW can also be estimated with single-shell data [13], however, to our knowledge, this type of validation study has not been performed in pathological white matter. Replication attempts with diffusion imaging sequences optimized for NODDI will be important. In addition, multishell imaging will also allow quantification of neurite density, which is not reliably quantifiable with single-shell data. To ameliorate susceptibility-induced artifacts, we used an anatomical reference image for co-registration. Five of the datasets were processed without the reference image, as described in the method section. It is, therefore, possible that we lost information in regions of signal compression in these datasets.

In conclusion, we demonstrated altered fiber complexity in medication-naïve and unmedicated patients with SZ. Lesser uniformity of fibers at the whole-brain level was predictive of subsequent poor response to antipsychotic treatment, suggesting this measure to be a clinically relevant biomarker. Interestingly, we found no significant changes in NODDI diffusion indices after short-term treatment with risperidone. Our data support that biophysical diffusion models have promise for the in vivo evaluation of brain microstructure in this devastating neuropsychiatric syndrome.

\section{FUNDING AND DISCLOSURE}

This work was supported by the National Institute of Mental Health (R01MH081014 and R01MH102951, ACL; K23MH106683, NVK). We would like to thank UAB IT Research Computing for providing the HPC resources (compute, storage, and networking) for this project. Cheaha is supported in part by the National Science Foundation under Grant no. OAC-1541310, the University of Alabama at Birmingham, and the Alabama Innovation Fund. $A C L$ has received an investigator initiated grant from Janssen Pharmaceuticals. The remaining authors declare no competing interests.

\section{ADDITIONAL INFORMATION}

Publisher's note: Springer Nature remains neutral with regard to jurisdictional claims in published maps and institutional affiliations.

\section{REFERENCES}

1. Kelly S, Jahanshad N, Zalesky A, Kochunov P, Agartz I, Alloza C, et al. Widespread white matter microstructural differences in schizophrenia across 4322 individuals: results from the ENIGMA Schizophrenia DTI Working Group. Mol Psychiatry. 2018:23:1261-9.

2. Bopp MHA, Zollner R, Jansen A, Dietsche B, Krug A, Kircher TTJ. White matter integrity and symptom dimensions of schizophrenia: a diffusion tensor imaging study. Schizophr Res. 2017;184:59-68.

3. Kochunov P, Rowland LM, Fieremans E, Veraart J, Jahanshad N, Eskandar G, et al. Diffusion-weighted imaging uncovers likely sources of processing-speed deficits in schizophrenia. Proc Natl Acad Sci USA. 2016;113:13504-9.

4. Skelly LR, Calhoun V, Meda SA, Kim J, Mathalon DH, Pearlson GD. Diffusion tensor imaging in schizophrenia: relationship to symptoms. Schizophr Res. 2008;98:157-62.

5. Wolkin A, Choi SJ, Szilagyi S, Sanfilipo M, Rotrosen JP, Lim KO. Inferior frontal white matter anisotropy and negative symptoms of schizophrenia: a diffusion tensor imaging study. Am J Psychiatry. 2003;160:572-4. 
6. Reis Marques T, Taylor H, Chaddock C, Dell'acqua F, Handley R, Reinders AA, et al. White matter integrity as a predictor of response to treatment in first episode psychosis. Brain. 2014;137(Pt 1):172-82.

7. Luck D, Buchy L, Czechowska Y, Bodnar M, Pike GB, Campbell JS, et al. Frontotemporal disconnectivity and clinical short-term outcome in first episode psychosis: a DTI-tractography study. J Psychiatr Res. 2011;45:369-77.

8. Mitelman SA, Newmark RE, Torosjan Y, Chu KW, Brickman AM, Haznedar MM, et al. White matter fractional anisotropy and outcome in schizophrenia. Schizophr Res. 2006;87:138-59.

9. Kubicki M, McCarley R, Westin CF, Park HJ, Maier S, Kikinis R, et al. A review of diffusion tensor imaging studies in schizophrenia. J Psychiatr Res. 2007;41:15-30.

10. Reid MA, White DM, Kraguljac NV, Lahti AC. A combined diffusion tensor imaging and magnetic resonance spectroscopy study of patients with schizophrenia. Schizophr Res. 2016;170:341-50.

11. Samartzis L, Dima D, Fusar-Poli $P$, Kyriakopoulos $M$. White matter alterations in early stages of schizophrenia: a systematic review of diffusion tensor imaging studies. J Neuroimaging. 2014;24:101-10.

12. Zhang $H$, Schneider $T$, Wheeler-Kingshott $C A$, Alexander DC. NODDI: practical in vivo neurite orientation dispersion and density imaging of the human brain. Neuroimage. 2012;61:1000-16.

13. Edwards L, Pine KJ, Ellerbrock I, Weiskopf N, Mohammadi S. NODDI-DTI: estimating neurite orientation and dispersion parameters from a diffusion tensor in healthy white matter. Front Neurosci. 2017;11:720.

14. Colgan N, Siow B, O'Callaghan JM, Harrison IF, Wells JA, Holmes HE, et al. Application of neurite orientation dispersion and density imaging (NODDI) to a tau pathology model of Alzheimer's disease. Neuroimage. 2016;125:739-44.

15. Grussu F, Schneider T, Tur C, Yates RL, Tachrount M, lanus A, et al. Neurite dispersion: a new marker of multiple sclerosis spinal cord pathology? Ann Clin Transl Neurol. 2017;4:663-79.

16. Sato K, Kerever A, Kamagata K, Tsuruta K, Irie R, Tagawa K, et al. Understanding microstructure of the brain by comparison of neurite orientation dispersion and density imaging (NODDI) with transparent mouse brain. Acta Radio Open. 2017;6:2058460117703816.

17. Schilling KG, Janve V, Gao Y, Stepniewska I, Landman BA, Anderson AW. Histological validation of diffusion MRI fiber orientation distributions and dispersion. Neuroimage. 2018;165:200-21.

18. Sykova E, Nicholson C. Diffusion in brain extracellular space. Physiol Rev. 2008;88:1277-340.

19. Fick HJ, Daianu M, Pizzolato M, Wassermann D, Jacobs RE, Thompson PM, et al. (2017). Comparison of biomarkers in transgenic alzheimer rats using multi-shell diffusio MRI. In: Fluster A, Gohosh A, Kaden E, Rathi Y, Reisert M, editors. Computational Diffusion MRI. Cambridge: Springer.

20. Rae CL, Davies G, Garfinkel SN, Gabel MC, Dowell NG, Cercignani M, et al. Deficits in neurite density underlie white matter structure abnormalities in first-episode psychosis. Biol Psychiatry. 2017;82:716-25.

21. Lyall AE, Pasternak O, Robinson DG, Newell D, Trampush JW, Gallego JA, et al. Greater extracellular free-water in first-episode psychosis predicts better neurocognitive functioning. Mol Psychiatry. 2018;24:701-7.

22. Oestreich LK, Pasternak O, Shenton ME, Kubicki M, Gong X, Australian Schizophrenia Research $B$, et al. Abnormal white matter microstructure and increased extracellular free-water in the cingulum bundle associated with delusions in chronic schizophrenia. Neuroimage Clin. 2016;12:405-14.

23. Szeszko PR, Robinson DG, Ikuta T, Peters BD, Gallego JA, Kane J, et al. White matter changes associated with antipsychotic treatment in first-episode psychosis. Neuropsychopharmacology. 2014;39:1324-31.

24. Carpenter WT Jr., Gold JM, Lahti AC, Queern CA, Conley RR, Bartko JJ, et al. Decisional capacity for informed consent in schizophrenia research. Arch Gen Psychiatry. 2000;57:533-8.

25. Pierpaoli C, Walker L, Irfanoglu MO, Barnett A, Basser P, Chang L-C, et al., editors. TORTOISE: An integrated software package for processing of diffusion MRI data. ISMRM 18th Annual Meeting, Stockholm, Sweden; 2010

26. Kraguljac NV, Anthony T, Skidmore FM, Marstrander JR, Morgan CJ, Reid M, et al. Micro- and macrostructural white matter integrity in never-treated and currently unmedicated patients with schizophrenia and effects of short term antipsychotic treatment. Biol Psychiatry. 2019a;4:462-71.

27. Yin J, Liu Y, Crosby LD, Anthony T, Burdyshaw C, Brook RG, et al., editors. Optimization of non-linear image registration in AFNI. XSEDE. Miami, Florida: ACM; 2016.

28. Theys C, Wouters J, Ghesquiere P. Diffusion tensor imaging and resting-state functional MRI-scanning in 5- and 6-year-old children: training protocol and motion assessment. PloS ONE. 2014;9:e94019.

29. Cox RW, Chen G, Glen DR, Reynolds RC, Taylor PA. fMRI clustering and falsepositive rates. Proc Natl Acad Sci USA. 2017a;114:E3370-E3371.

30. Cox RW, Chen G, Glen DR, Reynolds RC, Taylor PA. FMRI clustering in AFNI: falsepositive rates redux. Brain Connect. 2017b;7:152-71.
31. Hadley JA, Kraguljac NV, White DM, Ver Hoef L, Tabora J, Lahti AC. Change in brain network topology as a function of treatment response in schizophrenia: a longitudinal resting-state fMRI study using graph theory. NPJ Schizophr. 2016;2:16014.

32. Kraguljac NV, Morgan CJ, Reid MA, White DM, Jindal RD, Sivaraman S, et al. A longitudinal magnetic resonance spectroscopy study investigating effects of risperidone in the anterior cingulate cortex and hippocampus in schizophrenia. Schizophr Res. 2019b. https://doi.org/10.1016/j.schres.2018.12.028. [Epub ahead of print]

33. Kraguljac NV, White DM, Hadley JA, Visscher K, Knight D, ver Hoef L, et al. Abnormalities in large scale functional networks in unmedicated patients with schizophrenia and effects of risperidone. Neuroimage Clin. 2016;10:146-58.

34. Mollink J, Kleinnijenhuis M, Cappellen van Walsum AV, Sotiropoulos SN, Cottaar M, Mirfin C, et al. Evaluating fibre orientation dispersion in white matter: comparison of diffusion MRI, histology and polarized light imaging. Neuroimage. 2017;157:561-74.

35. Grazioplene RG, Bearden CE, Subotnik KL, Ventura J, Haut K, Nuechterlein KH, et al. Connectivity-enhanced diffusion analysis reveals white matter density disruptions in first episode and chronic schizophrenia. Neuroimage Clin. 2018;18:608-16.

36. Zhuo C, Ma X, Qu H, Wang L, Jia F, Wang C. Schizophrenia patients demonstrate both inter-voxel level and intra-voxel level white matter alterations. PLoS ONE. 2016;11:e0162656.

37. Brickman AM, Buchsbaum MS, Ivanov Z, Borod JC, Foldi NS, Hahn E, et al. Internal capsule size in good-outcome and poor-outcome schizophrenia. J Neuropsychiatry Clin Neurosci. 2006;18:364-76.

38. Mitelman SA, Torosjan $Y$, Newmark RE, Schneiderman JS, Chu KW, Brickman AM, et al. Internal capsule, corpus callosum and long associative fibers in good and poor outcome schizophrenia: a diffusion tensor imaging survey. Schizophr Res. 2007;92:211-24.

39. Rosenberger G, Nestor PG, Oh JS, Levitt JJ, Kindleman G, Bouix S, et al. Anterior limb of the internal capsule in schizophrenia: a diffusion tensor tractography study. Brain Imaging Behav. 2012;6:417-25.

40. Zhou SY, Suzuki M, Hagino $H$, Takahashi T, Kawasaki Y, Nohara S, et al. Decreased volume and increased asymmetry of the anterior limb of the internal capsule in patients with schizophrenia. Biol Psychiatry. 2003;54:427-36.

41. Walter M, Henning A, Grimm S, Schulte RF, Beck J, Dydak U, et al. The relationship between aberrant neuronal activation in the pregenual anterior cingulate, altered glutamatergic metabolism, and anhedonia in major depression. Arch Gen Psychiatry. 2009;66:478-86.

42. Bracht T, Schnell S, Federspiel A, Razavi N, Horn H, Strik W, et al. Altered corticobasal ganglia motor pathways reflect reduced volitional motor activity in schizophrenia. Schizophr Res. 2013;143:269-76.

43. Viher PV, Stegmayer K, Giezendanner S, Federspiel A, Bohlhalter S, Vanbellingen T, et al. Cerebral white matter structure is associated with DSM-5 schizophrenia symptom dimensions. Neuroimage Clin. 2016;12:93-99.

44. Walther S, Stegmayer K, Federspiel A, Bohlhalter S, Wiest R, Viher PV. Aberrant hyperconnectivity in the motor system at rest is linked to motor abnormalities in schizophrenia spectrum disorders. Schizophr Bull. 2017;43:982-92.

45. Buchsbaum MS, Schoenknecht $P$, Torosjan Y, Newmark R, Chu KW, Mitelman S, et al. Diffusion tensor imaging of frontal lobe white matter tracts in schizophrenia. Ann Gen Psychiatry. 2006;5:19.

46. Mamah D, Conturo TE, Harms MP, Akbudak E, Wang L, McMichael AR, et al. Anterior thalamic radiation integrity in schizophrenia: a diffusion-tensor imaging study. Psychiatry Res. 2010;183:144-50.

47. Ublinskii MV, Semenova NA, Lukovkina OV, Sidorin SV, Lebedeva IS, Kaleda VG, et al. Characteristics of diffusion in the corticospinal tract of patients with early stage of schizophrenia: diffusion tensor magnetic resonance imaging. Bull Exp Biol Med. 2015;159:29-31.

48. Liang S, Li Y, Zhang Z, Kong X, Wang Q, Deng W, et al. Classification of firstepisode schizophrenia using multimodal brain features: a combined structural and diffusion imaging study. Schizophr Bull. 2018. https://doi.org/10.1093/ schbul/sby091.

49. Douaud G, Mackay C, Andersson J, James S, Quested D, Ray MK, et al. Schizophrenia delays and alters maturation of the brain in adolescence. Brain. 2009;132 (Pt 9):2437-48. [Epub ahead of print]

50. Crossley NA, Marques TR, Taylor H, Chaddock C, Dell'Acqua F, Reinders AA, et al. Connectomic correlates of response to treatment in first-episode psychosis. Brain. 2017;140:487-96.

51. Oestreich LK, Lyall AE, Pasternak O, Kikinis Z, Newell DT, Savadjiev P, et al. Characterizing white matter changes in chronic schizophrenia: a free-water imaging multi-site study. Schizophr Res. 2017;189:153-61.

52. van Berckel BN, Bossong MG, Boellaard R, Kloet R, Schuitemaker A, Caspers E, et al. Microglia activation in recent-onset schizophrenia: a quantitative (R)-[11C]PK11195 positron emission tomography study. Biol Psychiatry. 2008;64:820-2. 
A longitudinal neurite and free water imaging study in patients with a... NV Kraguljac et al.

53. Doorduin J, de Vries EF, Willemsen AT, de Groot JC, Dierckx RA, Klein HC. Neuroinflammation in schizophrenia-related psychosis: a PET study. J Nucl Med. 2009;50:1801-7.

54. Kenk M, Selvanathan T, Rao N, Suridjan I, Rusjan P, Remington G, et al. Imaging neuroinflammation in gray and white matter in schizophrenia: an in-vivo PET study with [18F]-FEPPA. Schizophr Bull. 2015;41:85-93.

55. Takano A, Arakawa R, Ito H, Tateno A, Takahashi H, Matsumoto R, et al. Peripheral benzodiazepine receptors in patients with chronic schizophrenia: a PET study with [11C]DAA1106. Int J Neuropsychopharmacol. 2010;13:943-50.
56. Pasternak O, Westin CF, Bouix S, Seidman LJ, Goldstein JM, Woo TU, et al. Excessive extracellular volume reveals a neurodegenerative pattern in schizophrenia onset. J Neurosci. 2012;32:17365-72.

57. Pasternak O, Sochen N, Gur Y, Intrator N, Assaf Y. Free water elimination and mapping from diffusion MRI. Magn Reson Med. 2009;62:717-30.

58. Gallego JA, Robinson DG, Sevy SM, Napolitano B, McCormack J Lesser $\mathrm{ML}$, et al. Time to treatment response in first-episode schizophrenia: should acute treatment trials last several months? J Clin Psychiatry. 2011; 72:1691-6. 